\title{
Socio-historical knowledge: the experience of philosophical-epistemological reflection
}

\author{
Vasiliy Strelchenko ${ }^{1 *}$, Vitaliy Balakhonsky ${ }^{2}$, Maxim Bakhtin $^{3}$, Liudmila Balakhonskaya ${ }^{4}$, \\ and Evgenii Ivanov ${ }^{1}$ \\ ${ }^{1}$ Herzen State Pedagogical University of Russia, 197046, 26, Malaya Posadskaya str., Saint \\ Petersburg, Russia \\ ${ }^{2}$ St. Petersburg University of the Ministry of Internal Affairs of Russia, 198206, 1, Pilot Pilyutov str., \\ Saint-Petersburg, Russia \\ ${ }^{3}$ International Professorial Club (Italia) 97100, via Velardo, 35, Ragusa, Italia \\ ${ }^{4}$ St-Petersburg State University, School of Journalism and Mass Communications 191186, 7-9, \\ Universitetskaya naberezhnaya, St-Petersburg, Russia
}

\begin{abstract}
The fact that the principle of pluralism of opinions prevails in today's socio-historical knowledge shows that the "postmodern condition" (J-F. Lyotard), which began in the late 70s and early 80 s of the twentieth century, expresses the characteristic and most significant features of its epistemological situation. This situation is characterized by a fundamental change of emphasis in the approach itself, not even to the solution, but to the very formulation of the problem of the truth values and the conditions for the reliability of the statements put forward. In the recent past, it was almost generally accepted to consider the issues of reliability of sociohistorical knowledge as essentially methodological and to associate the possibility of understanding the truth with the improvement of the system of instruments of the scientific method, then in recent decades, the situation has radically changed. Under the influence of strengthening the position of the "postmodern condition", we are no longer talking about criterion (methodological, logical, epistemological, etc.), but about the meaning of truth in general, and socio-historical truth in particular.
\end{abstract}

\section{Introduction}

Knowledge in general, and in its socio-historical representations, in particular, reveals in the twentieth and at the beginning of the twenty-first century obvious features of the loss of certainty due to the almost insurmountable difficulties of interpreting truth values. Under the influence of arguments in favor of the historicity of scientific knowledge (German classics, first positivism, Marxism, postpositivism, etc.), the crisis of the foundations of mathematical natural science at the beginning of the twentieth century, the loss of logic and mathematics of the meaning of universal languages for describing and strengthening the ideas of a plurality of types of rationality, the modern epistemological situation in sociohistorical knowledge is characterized by almost boundless pluralism of opinions [1]. The

* Corresponding author: universdoktor@gmail.com 
existing projects to overcome skepticism, relativism, and uncertainty by "modernizing" the historical (neo-Marxism, neo-Pragmatism, neoliberalism, etc.) and putting forward the latest doctrinal "inventions" of socio-historical rationality (information society, knowledge society, multiculturalism, etc.) serve the purpose rather not solving, but even more actualizing the problems of the nature of social subjectivity, social theory and its subject, historical fact, methods and logic of socio-historical cognition, and, finally, - the conditions of its reliability and truth [2].

The extension of the principle of "pluralism of opinions" to the field of assessing the conditions for identifying reliable socio-historical knowledge led to the formation of beliefs about the limitations, epistemological neutrality, the multiplicity of truth, which includes almost a whole "family" of non-classical forms (pragmatic, linguistic, existentialhermeneutic, etc.).

A paradoxical situation has developed: since the classical concept of truth does not meet the challenges of today's scientific search, the concept of truth itself is regarded as having no significant epistemological significance. In the philosophy and methodology of science of recent decades, the concept of truth, which previously defined the theoretical and practical value and goals of scientific knowledge, is not only rarely used but is also subjected to harsh criticism as a sign of mythologization, as the main obstacle on the path of human knowledge, which need to be deconstructed. And the point here, first of all, is that objective reality, or " things as they exist "in themselves", are not directly given to us, and in each particular case, we are talking about the correspondence of socio-historical (or natural-historical) knowledge to experimentally established empirical facts.

The fundamental contradictions that have arisen on this basis between the traditional beliefs about the unity (and uniqueness) of truth, on the one hand, and the new ideas about its multiplicity, on the other, mark the beginning of a whole revolution in the methodology and philosophy of science.

In contrast to the previous tradition, nowadays on the fore promote not the issues of improving the technical (methodological, logical, etc.) identification of truth values but the problem of the nature of truth [3].

The tasks that arise from this have not local, but of world-historical significance and marking the beginning of fundamental qualitative reconstructions of worldview systems and social practices. Because of the uncertainty of the concept of truth, the spiritual situation of our time is subject to the same large-scale crisis as the religious worldview of the Greco-Roman world at the origins of Christianity.

\section{Materials and method}

Under the influence of the consequences of linguistic, epistemological, ontological, communicative turns, ideas of logical empiricism and analytic philosophy in the philosophy of the twentieth and early twenty-first centuries, the classical concept of truth is developed in the form of a complex of problems such as the correspondence of "ideas" to sensory data( empiricism), judgments to their subject, statements to established facts (B. Russell, L. Wittgenstein, G. Moore), the provisions of the theory to its empirical basis (M. Schlick, K. Popper), objective reality based on the verification of socio-historical practice (Marxism), etc. [4]. The reduction of the problem of truth to the properties of sign-symbolic systems and linguistic expressions is the main reason for the refusal of many of the influential modern philosophical trends to use the classical concept of truth in the characterization of scientific and philosophical knowledge (philosophy of life, structuralism, postmodernism, existentialism, etc.). According to J. Derrida, R. Rorty, P. Feyerabend [5] the notion of truth is outdated, meaningless, ideologically loaded [6]. As a result of the attempts made to overcome the difficulties encountered in substantiating the statements of mathematical 
natural science and socio-humanitarian sciences, three main non-classical concepts of truth have emerged, which constitute the opposition to the classical one. Now, along with the theories of correspondence and coherence, the pragmatic (C. Peirce, W. James, etc.), linguistic (L. Wittgenstein, B. Russell, M. Schlick, etc.) and existential-hermeneutic (M. Heidegger) concepts of truth are among the most generally accepted [7].

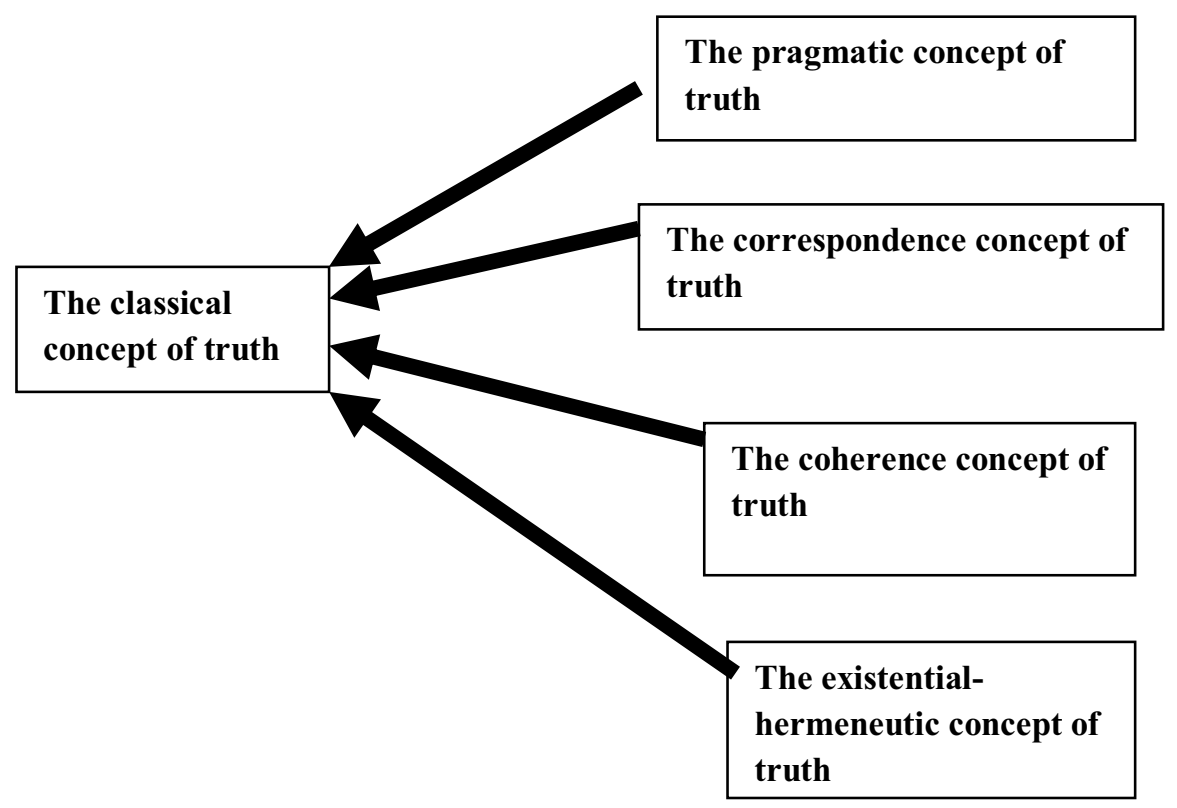

Fig. 1. The classical concept of truth and its alternatives.

In contrast to the classical one, the pragmatic concept of truth is defined in the main points of its content in the philosophy of F. Nietzsche, specially developed and developed by epistemological studies of American pragmatism (C. Pearce, W. James, J. Dewey).

According to F. Nietzsche, all metaphysical systems, including science, are intellectual fictions, devoid of a referential basis, conceptual constructions that express certain human interests. Rejecting the referential meaning of empirical fact as a derivative of theory, F. Nietzsche emphasizes that true knowledge is useful for life, life-affirming. Such knowledge is true not because of an adequate reflection of the natural or social reality, but because of the effective service of life.

The idea of the vital and practical utility of knowledge as a criterion of its truth is the basis of the variety of pragmatistic epistemological views. According to C. Peirce, for example, truth is a property of the process of practical action unfolded in time, which simultaneously reveals both existential-ontological and actually rational-logical properties. This is not about confirming or certifying the truth, but about creating it, actively shaping it through practical actions. Knowledge becomes true only insofar as it helps us to come into a satisfactory relation to other parts of our experience. After all, any idea is useful insofar as it is true, and true in so far as it is useful.

It should be emphasized here that the pragmatist version of the truth serves not only as a "harmonization of the idea with reality" and as a way to organically include the subject in the sphere of life practice. Truth also serves as a formative principle of social relations. The "exchange of truths" reveals the obvious features of a specific social connection that integrates individual human individuals into productive social communities. However, due to the initial attitudes of radical empiricism and the consistent principle of pluralism, they 
are associations of extremely atomized individuals, for whom any form of collective organization has the right to exist only as environmental conditions and as a tool for achieving purely selfish interests [7].

As in the past, in the era of American pragmatist "patristics" (C. Pierce, W. James, J. Dewey), and now, at the origins of the XXI century, realists and anti-realists, analytical and non-analytical philosophers, modernists and postmodernists call themselves pragmatists. Despite significant differences, they still share a common focus on issues of realism, justification of knowledge, and truth. A century later, after the theoretical affirmation of the oppositions of James '" nominalism "and Peirce's" realism", the discussion of the problem of truth continues in the horizon of their ontological and theoretical-cognitive attitudes. The magic and propaganda "charm" of the term "pragmatism" is now so great that positioning yourself as a "pragmatic politician"," pragmatic economist", "pragmatic manager", "pragmatic teacher", etc. has become an important element of the strategy of introducing into the public consciousness (as well as individual) beliefs not only in justification but also justice, the enduring anthropological and social value of any, even the most fantastic and ridiculous" business project".

The existential-hermeneutical concept of truth is presented in its most developed form in M. Heidegger's fundamental ontology. The concept of truth in Heidegger and his followers is based on an existential-ontological interpretation of the "historicity" of human existence. Truth is an expression of the "authenticity" of human existence and is understood as an "event" of life practice. It does not belong to a person, but belongs to him, expressing the properties of his individuality. The ontological distinction between Being and being, and hence between philosophy as the science of Being and the positive sciences as the sciences of being, leads to the conclusion that scientific knowledge has at its core the character of detection, which means that it deals only with the objectified manifestation of truth, with ontic truth (being), and not ontological (Being). Being and the truths of Being are beyond the reach of the objectifying thinking of scientific rationality. At the same time, due to the universalization of its methods (objectification), knowledge about the structures of existence is given the importance of the truths of Being. Invading the transcendental domain of ontology, scientific, i.e., objectifying thinking, takes the path of false interpretations and total delusions generated by the indistinguishability of ontic and ontological truths. The prospect of their separation and identification is associated with the "optics" of the phenomenological method, which allowed E. Husserl to explain the crisis of the European sciences by "objectifying reification", the naturalization of their conceptual, terminological means and ideal objects [8].

Heidegger's idea of truth unfolds in the ontological horizon and is historically and genetically linked to the pre-Socratic intellectual tradition. Heidegger is not talking about its literal reanimation, but about the reconstruction of the lost strategies of understanding the truth as the "unseen", "uncovered" existence. Understanding is considered both as a form of the authenticity of human existence and as a way of understanding its true meaning, which is revealed in the connection with Being as such. Hence the conclusion about the identity of truth and the meaning of Being, as well as the focus on the problem of "events", which include questions of the ontological conditions of the very possibility of the conjugacy of Being and being of man. Truth is not a property of a logically correctly constructed sentence, nor is it its correspondence to an out-of-place subject to an object it is a way of releasing the being, a release within which human freedom is realized. The goal of fundamental ontology is to overcome the empty metaphysical abstractions of truth and to reveal the meaning of Being, which is not identical with being. 


\section{Discussion}

The breakthrough to the truth as the authenticity of human existence is a hermeneutical process that unfolds at a deeper level than hypothetical-deductive, rational-logical research. The procedures of hermeneutical meaningfulness, or, what is the same thing, intuitive explication of meaning, are realized in the emotionally intense field of psychological experiences of the individual and are incompatible with the attitudes of the New European discourse on antipsychologism and objectivity. In the context of the uncertainty of existing ideas about the truth and the reduction of the tasks of its identification to the analysis of the meaning of language expressions, it is the language that acquires the meaning of the primary, initial reality, and the forms of its logical and grammatical organization are realized as completely independent entities, neutral in relation to the external in relations with consciousness objects of nature and society.

In its most "extremist" version, this position is expressed by the well-known maxim that reads: "It is not a man who speaks of himself through language, but a language that speaks of itself through man." However, the failure of the attempts made on this path to eliminate "metaphysics" by means of logical and mathematical analysis (logical empiricism) and modeling the language of science with the help of the developed artificial languages (C. Hempel, R. Carnap, etc.) motivated the beginning of the transition to the interpretation of the statements of theoretical natural science and mathematics in terms of ordinary, or natural language. So, according to K. Jaspers, several features can be distinguished in historical consciousness: first, it makes a request for the comprehensiveness and accuracy of research methods, for the understanding and objectification of causal factors in noncausal categories, second, historical consciousness begins to overcome the picture of history as a complete whole, third, the value of the individual and unique is realized, fourth, history becomes a problem of understanding the present and ceases to be an object of aesthetic pleasure, fifth, humanity begins to be understood as something concrete, in relation to which we can talk about the meaning and structure of history, sixth, the main feature of historical consciousness is the indissolubility of history and the present, the impossibility of "leaving" the present in the past, the impossibility of forgetting the past, but the deepening of the" now " consciousness of the past and the future [9]. Some of these characteristics arise in the historical consciousness together with its emergence in the "axial time", some are new. Since for existentialism consciousness is "real and true" [9], and history is the most important reality of self-identification, historical consciousness turns out to be the consciousness of a person of our time in general - existence is historical. Its transcendental basis in Jasper's opinion is the unconscious, located in the space of everyday life. That basis carries the essential characteristics that were listed above. Turning to everyday life as the ultimate foundation of rationality and the products of reflective thinking in general means entering the space of antinomically fixed oppositions of physics and metaphysics, rational and empirical, natural and artificial, theory and experience, intuitive and logical-discursive, ethics and logic, everyday and non-everyday, traditionalism and innovation, explanation and understanding, etc.

Taking into account their social contexts and the already clarified trends of spiritual evolution, it is impossible not to assume that the scale of differences and the boundaries of the identity of the phenomena of everyday consciousness and the structures of reflective thinking generally coincide with the" lines "of demarcation of "naturally arisen" and "artificially created" formations of subject-practical experience.

In fact, the removal of the opposition between the natural and the artificial, the identification of the artificial with the natural, is an event that marks a turning point in the history of not only philosophy and science but also of European humanity as a whole. It is enough to point out that the transformation of the artificial into the natural did not eliminate 
the centuries-old tradition of giving a fundamentally important role to account for these differences for understanding the natural organization and development of society, in the life of which the spheres of culture (natural) and civilization (artificial) were identified.

The existence of reflexive and non-reflexive "layers" of consciousness, the opposition of the sensory-visual experience of everyday life and the products of scientific and theoretical construction, the attitudes of traditionalism and innovation, cognitive strategies of artistic-figurative and logical-conceptual thinking, alternatives to intuitive and logicaldiscursive explanation and understanding, ethics and logic, natural and artificial languages, etc. they are an expression of the conflict of " naturally occurring "and" created by civilization " components in the structures of both cognitive and socio-historical activities.

\section{Results}

Everyday consciousness as a form of meaningfulness of the experience of everyday life is oriented by well-defined value-goal settings. Meanwhile, mathematical natural science, modern technoscience, and the corpus of socio-humanitarian sciences do not contain any recommendations about the anthropological and cultural-civilizational consequences of applying their own achievements [10]. Due to the obvious dependence of their use on the arbitrariness of various kinds of subjective interests (individual or group), there is now an increasingly acute awareness of the need to rely on axiologically verified linguistically correct and confirmed by the data of sensory perception, opaque to reflective thinking truths of common sense. They include, as is well known, the totality of generally accepted rules of behavior, principles, beliefs, and assessments, recipes for action, etc., that make up the content of the universal tradition. The socio-historical tradition is a" mechanism " for the transmission of knowledge, and skills proven by the experience of generations, the truth values of which are determined by references to authorities, historical precedents, or the latest results of scientific research. The intellectual tradition and the experience of everyday life were elevated to the rank of an authority that sanctioned any knowledge, including socio-historical knowledge, on the part of its validity, reliability, and truth. The way out of the dead ends of the indistinguishability of truth and falsehood is increasingly seen in the ways of dialogue with tradition as an independent source of knowledge and as a means of elimination metaphysics. Emphasized the limitations of a narrowly epistemological interpretation of knowledge, which arises not as a result of theoretical and cognitive operations, but as a result of experiencing the "experience of consciousness"," life experience"," historical experience"," experience of generations"," experience of the world $"$ in the unity of their rational-theoretical and sensory-objective dimensions. However, the mere recognition of the cognitive value of "life" or "historical" experience, the experience of "intellectual tradition", etc., does not yet prejudge the answers to questions about the nature of the underlying type of rationality as a formative principle of everyday life. This situation is largely clarified thanks to the concept of the "lifeworld" systematically developed by E. Husserl [11], techniques for expressing objectivity in acts of consciousness, and the theory of language games put forward by L. Wittgenstein.

According to A. Schutz, a proponent of the idea of the identity of the "lifeworld" "and everyday life, the everyday world is intersubjective because we live in it as people among other people it is the world of culture because from the very beginning the lifeworld is for us a universe of designations, that is, a semantic network that we must interpret and a world of semantic relationships that we establish only through our actions in this world. 

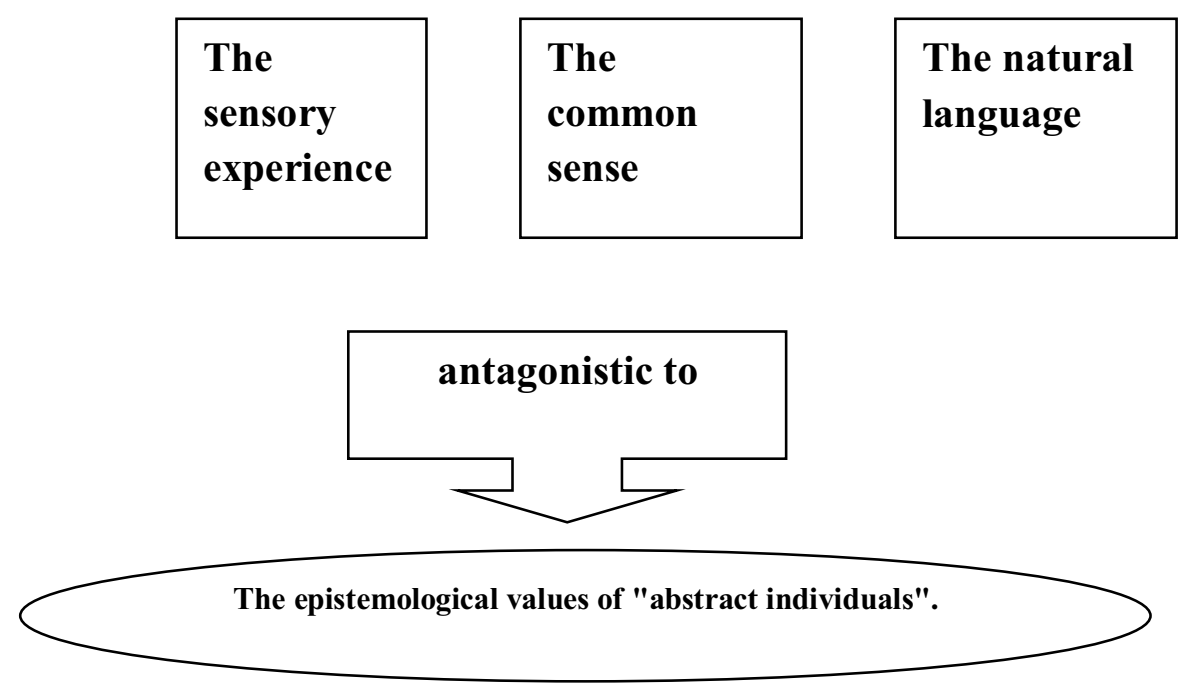

Fig. 2. The "lifeworld" and its cognitive structures.

The "lifeworld" and its cognitive structures (sensory experience, common sense, natural language) not only do not coincide but are also antagonistic to the epistemological values of "abstract individuals". The distance between the subjectivity of the lifeworld and the "objective true world» lies in the fact that the latter is a theoretical-logical substructure that encompasses what is fundamentally not perceived in experience while the subjective of the lifeworld is marked by experiential cognoscibility. Institutions and epistemological initiatives of big science, philosophy, religion, art, technology, etc. which grown from lifeworld although they are located in the space of the intersubjective world, they as well as the lifeworld are constituted by the intentional acts of consciousness. The complementarity of the action of the structures of intentional activity of consciousness can be considered as an immanent experience of everyday life "mechanism" of rationalization, which is an epistemological opposition to the techniques of reflective thinking. Its artificial, specialized scientific and theoretical constructions, developed based on civilizational progress, are characterized, as can be observed, by an ever-increasing anthropological aggressiveness [12]. Meanwhile, attempts to verify them using both logical-mathematical and experimental criteria do not achieve the goal.

Overcoming the threat of the "disintegration of the structures of reflective thinking" (M. Heidegger) is largely due to the results of research on the relationships between the natural languages of everyday experience and the artificial, specialized languages of modern science developed in the concept of linguistic games by L. Wittgenstein. However, the extrapolation of its statements to the field of everyday experience is fraught with the disintegration of its characteristic type of rationalization due to the variety of rules of language games.

The interpretation of artificial, anthropologically neutral "dictionaries" of mathematical natural science and socio - humanitarian sciences in the natural languages of everyday life is only an apparently impossible task. In the context of the historical approach, that is, transformed on the basis of the requirements of the principle of historicism, it loses the appearance of absolute insolubility and turns into a question of choosing the "material" for interpretation. In recent years, hopes for solving the problems of determining the conditions 
for the truth of historical knowledge are increasingly associated with research in the field of social epistemology. Despite the lack of consensus on the subject and the range of problems of social epistemology, it serves as an expression of the intentions of the "breakthrough" of non-classical rationality to the transcendence of history and historical consciousness. However, the very first steps of the movement in this direction are marked by the separation of positions in their relation to the reliability and truth of historical knowledge. A. Goldman, for example, considers truth in terms of the theory of correspondence, in connection with which knowledge is understood as a true belief. Along with this, nonclassical approaches were also defined in social epistemology, represented by the "strong program" (B. Barnes, D. Bloor), postmodernism (R. Rorty, J. Derrida, M. Foucault, etc.), and social constructivism (B. Latour, S. Woolgar) [13].

Here we can draw the following conclusion: the modern historical consciousness realizes itself in two modes. First, it can manifest itself as a classical historical consciousness, dating back to the Renaissance and perceived by us in the tradition of historical research at the turn of the XIX-XX century, and secondly, it exists in the form of a pseudo-historical consciousness, reproducing the formal structure of its classical prototype, but operating not with knowledge, but with pseudo-knowledge. The study and understanding of this duality of historical consciousness is a task for several disciplines: for history itself, for the philosophy of history, for the sociology of knowledge, and, as it seems, for social epistemology.

The formation of the methodology and epistemology of history is very accurately presented in the classic work of R. J. Collingwood "The Idea of history" [14]. Since its publication, history as a science has not only preserved but also developed its methodological and epistemological potential, which is confirmed by the growing number of studies, questions of the subject and method of historical knowledge, a significant expansion of its disciplinary space, and the focus on the problems of socio-historical subjectivity.

The matrices of historical knowledge and historical consciousness do not coincide. History as a scientific discipline and as a form of not only scientific but also pre-scientific and extra-scientific historical consciousness exists in the form of a variety of forms of perception of socio-historical time. These include a variety of traditional beliefs and stereotypes, local and interregional legends and myths, common sense judgments, historical narratives, utopian ideologies, popular history, pseudo-history, etc. The more diverse the composition of competing forms of historical knowledge, the higher the probability of its construction following the requirements of reliability and truth. The differences in the modes of manifestation of historical consciousness can be explained by one or another combination of its qualitative and quantitative characteristics. By classifying and systematizing them in one way or another, one can obtain a matrix of possible research into historical consciousness. However, the question arises, whether such research will be in the competence of social epistemology? According to S. Fuller, social epistemology is an interdisciplinary intellectual movement that tries to reformulate the problems of epistemology, considering knowledge as essentially socially conditioned. Humanitarian knowledge is considered by social epistemology theorists as an inseparable unity of humanitarian research and its social context. For the natural and social sciences, the possibility of differentiating the subject, the result of research, and the social context that influences them in the course of epistemological criticism are quite achievable. However, for historical or philosophical knowledge, a distraction from the social context is equivalent to the elimination of this knowledge altogether. And this leads to the need to recognize historical knowledge as belonging to a special philosophical-epistemological tradition peculiar only to it, which grows out of the experience of cognitive practices of the narratological sequence and, therefore, perhaps quite rightly referred to as social. 
The research of historians (with the rare exceptions of purely demographic or economic history) cannot be presented in the discursive form characteristic of the natural sciences. The result of historical research is the construction of a historical narrative since historical research includes at the same time the process of narration about it. The difference between a historical narrative and a fictional one is the nature of the reference. A fictional narrative is a reflection of a system of linguistic differences, whereas a historical narrative presupposes a representation of historical reality. Hence the differences between explanatory procedures and ideal objects. The historian cannot, unlike the writer, be satisfied with the internal coherence and completeness of events within the framework of verbal and terminological construction. He must relate it to the set of strictly established facts and justify the way they are connected.

It is well known that narratives are created in the form of imitation of practical action (Aristotle). In other words, the creation of a narrative relies on the structures of everyday thinking and the cognitive resources of common sense. First, to create a narrative, it is necessary to distinguish the intelligible structure of action from the physical movement in terms of purpose, motive, circumstances, interactions, etc. Such terms are implicitly contained in the structure of common sense in a non-reflexive form as derivatives of ontological understanding.

Secondly, the understanding of an action can only be realized on the basis of its symbolic mediations, fixed in everyday experience. The articulation of the meanings of such actions is carried out in the context of relevant symbolic systems. The symbolic system of everyday experience is fixed in the structure of everyday language and thus sets the context of description for individual actions, reduces the unique uniqueness of a particular action to the intersubjective meanings of everyday language. The system of symbolic expression of action should not be confused with the conceptual apparatus of sociology, anthropology, or history. In the social sciences, the articulation of the meaning of an action is defined by specially created categories, concepts, nomological explanations, etc., which cannot be mixed up with symbolic constructs in which culture distinguishes itself.

Third, the articulations of action in the structures of everyday language are temporary. The temporal nature of human experience is related to the narrative organization in such a way that human time is articulated in the narrative.

To imitate or represent an action, therefore, "...means first of all to distinguish what is in it from human action: its semantics, its symbolism, its temporality" [15]. The narrative, despite the fact that it makes a break with a concrete action, configures the abstract features that are contained in every human action.

It seems that the relationship between the narrative and the practical understanding of action has a dual character. On the one hand, the terms in which the understanding of the action is carried out must precede the construction of the narrative, on the other hand, the construction of the narrative gives these terms unity and reality.

\section{Conclusions}

Nevertheless, our practical understanding of action constitutes a narrative competence. It is important that the narrative, imitating life, can make intelligible (at the cost of reduction) situations that are not intelligible in everyday life experience. We can hope that the emergence of social epistemology as a philosophical discipline opens up the prospect of constructing an epistemology of history, as well as identifying historical, pseudo-historical, and extra-historical knowledge as products of the historical consciousness of the epoch. Apparently, social epistemology is an expression of the aspirations of liberation from metaphysics, which are also extended to historical thinking to interpret its forms in direct 
correlation with the features of the corresponding socio-cultural context. The implementation of such work is all the more important because up to the present time there is only no knowledge, but also a strict statement of the question about the nature of the philosophical and epistemological prerequisites for a synthetic, holistic vision of the object of socio-historical knowledge [16]. The special relevance of the issues of the epistemology of history is dictated by the necessity of understanding the essence of social changes in the "transition period" in the development of Russian society. Due to the scale and unprecedented nature of the processes of changing the social system, their connection with the progress of information technologies and globalization transformations, the solution of the problems of identifying reliable socio-historical knowledge affects the "physical balance" of the very existence of national culture, affects all aspects of human life and the development of society without exception.

\section{References}

1. L.V. Balakhonskaya, V.I. Strelchenko, V.V. Balakhonsky, T.A. Sadretdinova, I.V. Beresneva, Communicative strategy of discrediting opponents in the Russian political blogosphere, 2020 IEEE Communication Strategies In Digital Society Seminar, COMSDS 2020 Proceedings (2020)

2. V. Strelchenko, Proceedings of the A. I. Herzen Russian State Pedagogical University, 175, 105-115 (2015)

3. J. Baudrillard, Simulacra and simulations (Moscow, 2017) [Electronic resource] https://ru.bookmate.com/reader/Ieb6iyez?resource=book (accessed: 27.03.2021)

4. V. V. Balakhonsky, D. O. Shipunova, V. I. By Strelchenko, L. V. Balakhonskaya, Iu.V. Beresneva, International journal of criminology and sociology, 9, 941-949 (2020)

5. P. Feyerabend, Science in a free society (M.: Kanon, 2011) [Electronic resource] http://realfaq.ru/uploads/epistemologicheskij-anarhizm/txt19.htm (accessed: 27.03.2021)

6. V. Balakhonsky, M. Bakhtin, V. Strelchenko, Models and philosophicalepistemological representations of history (Moscow: Enciklopedist-Maxim, 2017)

7. V.A. Lectorskiy, E.O. Trufanova, Man, 30, 1, 102-124 (2019) [Electronic resource] https://ras.jes.su/chelovek/s023620070003025-4-1 (accessed: 27.03.2021).

8. L.A. Nikiforov, Epistemology \& philosophy of science, 56, 3, 20-29 (2019) [Electronic resource] https://www.elibrary.ru/item.asp?id=41258730 (accessed: 27.03 .2021 )

9. I.B. Mikirtumov, Philosophical Sciences, 1, 85-94 (2017)

10. S. Fuller, Epistemology \& philosophy of science 57, 4, 70-75 (2020) 